\title{
Does pterygopalatine canal injection with local anaesthetic and adrenaline decrease bleeding during functional endoscopic sinus surgery?
}

\author{
C J VALDES ${ }^{1}$, Y AL BADAAI ${ }^{2}$, M BOGADO ${ }^{1}$, M SAMAHA $^{3}$ \\ ${ }^{1}$ Department of Otolaryngology - Head and Neck Surgery, Hospital del Salvador, Universidad de Chile, Santiago, \\ Chile, ${ }^{2}$ Division of Otolaryngology - Head and Neck Surgery, Department of Surgery, Sultan Qaboos University \\ Hospital, Muscat, Oman, and ${ }^{3}$ Department of Otolaryngology - Head and Neck Surgery, Royal Victoria Hospital, \\ McGill University Health Centre, Montreal, Quebec, Canada
}

\begin{abstract}
Objective: To determine the effect of pterygopalatine fossa injection with xylocaine and adrenaline on: surgical field bleeding and blood loss during functional endoscopic sinus surgery for chronic rhinosinusitis, and the duration of the procedure.

Methods: A prospective, single-blinded, controlled trial was performed in a tertiary care academic centre. A total of 45 patients undergoing functional endoscopic sinus surgery for chronic rhinosinusitis, whose disease was symmetrical based on computed tomography grading, were included. A unilateral pterygopalatine fossa injection with 1 per cent xylocaine and 1:100 000 adrenaline was performed after the induction of anaesthesia. The contralateral side served as the control. The operating surgeon, who was blinded to the injected side, assessed the surgical field using a validated six-item grading system. Blood loss, blood pressure, heart rate and end-tidal carbon dioxide were recorded every 15 minutes for each side separately, and duration of surgery was noted.

Results: There was no statistically significant difference in the surgical field grade between the injected and noninjected sides $(p=0.161)$. There were no differences in blood loss or duration of surgery.

Conclusion: Pterygopalatine fossa injection prior to functional endoscopic sinus surgery did not decrease intraoperative surgical field bleeding, blood loss or duration of surgery.
\end{abstract}

Key words: Nasal Surgical Procedures; Hemorrhage; Pterygopalatine Fossa; Vasoconstrictor Agents

\section{Introduction}

Functional endoscopic sinus surgery (FESS) is the treatment of choice for chronic rhinosinusitis when maximal medical treatment fails. Control of bleeding during FESS is of primary importance for a safe and effective procedure, because mucosal bleeding may compromise visualisation of the intranasal anatomy. This could increase the risk of complications such as skull base injury with cerebrospinal fluid leak or intracranial injury, or penetration of the orbit with possible damage to the extra-ocular muscles and the optic nerve. ${ }^{1,2}$ In addition, more tissue damage to healthy mucosa is likely to occur in poor visibility conditions, and this can affect the development of post-operative synechiae which may re-obstruct sinus ostia. In addition to optimising surgical safety and minimising intra-operative and post-operative complications, a dry surgical field with better visualisation during
FESS will also reduce operative times and shorten post-operative convalescence.

Various methods have been advocated to reduce intra-operative bleeding during FESS. These include: elevation of the patient's head to reduce venous return; ${ }^{3}$ the use of bipolar cautery and powered instruments such as the microdebrider; premedication with beta blockers; a controlled hypotensive anaesthesia technique; and total intravenous anaesthesia. ${ }^{3-8}$ Despite these measures, some patients may still have a significant amount of bleeding intra-operatively.

While injection of local anaesthetic with adrenaline into the nasal mucosa is routinely performed at the start of the procedure, the injection of this agent into the greater palatine canal to induce vasoconstriction of the sphenopalatine artery is not widely adopted by sinus surgeons. This study aimed to assess the effect of greater palatine canal injection with xylocaine and 
adrenaline on intra-operative surgical field bleeding and overall blood loss during FESS.

\section{Materials and methods}

\section{Study design}

A prospective, single-blinded, controlled study was conducted. A sample size calculation was performed with a power of 80 per cent and a clinically significant difference in bleeding (Table I) between the sides of one point (standard deviation $(\mathrm{SD})=1$ ), with a significance level of 5 per cent $(p<0.05)$. For a paired design in which each subject serves as his or her own control, the calculation revealed that 45 patients were required. Computer-generated randomisation was used to determine the side on which the patient would receive the pterygopalatine fossa injection.

\section{Patients}

Approval to conduct the study was obtained from the McGill University Health Centre Research Ethics Committee. Patients older than 18 years, who were to undergo FESS carried out by the senior author (MS) for chronic sinusitis with or without nasal polyps, were invited to participate.

Disease symmetry between the right and left side was determined on pre-operative computed tomography (CT) scans using the Lund-Mackay CT grading system. ${ }^{9}$ Patients with a difference of more than three points between sides were considered to have asymmetric disease and were excluded.

Other exclusion criteria were: systemic corticosteroid use in the four weeks preceding surgery; untreated or poorly controlled hypertension; history of bleeding diathesis; and use of aspirin, anticoagulant or natural herbal substances in the four weeks prior to surgery.

\section{Operative technique}

After induction of general anaesthesia, the greater palatine foramen was identified according to its known landmarks in the hard palate. A 25 gauge, $6.35 \mathrm{~cm}$ (2.5 inch) needle was inserted in the greater palatine canal at a depth of $25 \mathrm{~mm}$ and an angle of 45

\begin{tabular}{|c|c|}
\hline \multicolumn{2}{|r|}{ 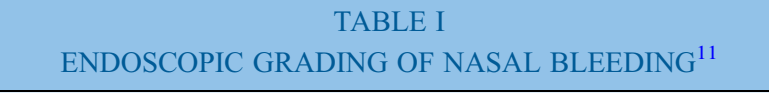 } \\
\hline Grade & Description \\
\hline 0 & No bleeding (cadaveric conditions) \\
\hline 1 & Slight bleeding - no suctioning required \\
\hline 2 & Slight bleeding - occasional suctioning required \\
\hline 3 & $\begin{array}{l}\text { Slight bleeding - frequent suctioning required; bleeding } \\
\text { threatens surgical field a few seconds after suction is } \\
\text { removed }\end{array}$ \\
\hline 4 & $\begin{array}{l}\text { Moderate bleeding - frequent suctioning required; } \\
\text { bleeding threatens surgical field directly after suction } \\
\text { is removed }\end{array}$ \\
\hline 5 & $\begin{array}{l}\text { Severe bleeding - constant suctioning required; surgical } \\
\text { field severely threatened (bleeding appears faster than } \\
\text { it can be removed by suction) \& surgery usually is not } \\
\text { possible }\end{array}$ \\
\hline
\end{tabular}

degrees. ${ }^{10}$ After aspiration to exclude intravascular penetration, $2 \mathrm{ml}$ of xylocaine 1 per cent with 1:100 000 adrenaline was slowly injected. The other side was not injected and served as the control. All injections were performed by the co-investigators (CJV or YAB) using the same technique. The surgeon evaluating the bleeding (MS) was not present at the time of injection and was therefore blinded to the side of injection. Oxymetazoline nasal spray was then applied intranasally, bilaterally. Approximately $6-7 \mathrm{ml}$ of 1 per cent xylocaine with 1:100 000 adrenaline was then used to infiltrate the lateral nasal wall mucosa bilaterally. Six pledgets soaked in a total of $5 \mathrm{ml}$ of 4 per cent cocaine hydrochloride solution were applied topically in the middle meatus region bilaterally, three on each side. The injection and topical cocaine were left to work for 7-8 minutes and surgery commenced.

The operating surgeon estimated the intensity of bleeding in the operative field every 15 minutes, according to the 6-point scale used by Boezaart et al. (Table I). ${ }^{11}$ At each assessment, blood pressure, heart rate (HR) and end-tidal carbon dioxide $\left(\mathrm{CO}_{2}\right)$ were recorded.

Surgery on the first side was completed prior to commencing surgery on the second side. All blood lost was collected and total blood loss was recorded separately for each side. In order to maximise the accuracy of blood loss measurement, all irrigation fluids, including the saline in the intravenous bag and tubing used for microdebrider irrigation, were collected at the conclusion of surgery on the first side and accounted for. A new intravenous saline bag was then installed and used for the second side.

\section{Statistical analysis}

Comparison of the surgical field grades between the injected and control sides for each subject was made using a Wilcoxon signed rank test. Total blood loss per side was compared within subjects using a $t$-distribution with a 95 per cent confidence interval (CI). As all of these values were measured over time, the averages per patient over time were used as the main outcome measures.

Finally, a linear regression was performed, with surgical field grade as the outcome variable, and HR, mean arterial pressure or end-tidal $\mathrm{CO}_{2}$ as covariates. Again, as these measures were taken over time, a repeated measures analysis was used to assess the correlation of these items over time.

\section{Results}

A total of 45 patients were included in the study: 30 males (66.7 per cent) and 15 females (33.3 per cent). The mean age \pm SD was $48.84 \pm 11.98$ years (range, 29-74 years). The mean Lund-Mackay score was $17.31(\mathrm{SD}=6.12)$ and mean surgery duration was 76.67 minutes $(95$ per cent $\mathrm{CI}=65.2-88.1$ ).

A comparison between injected and non-injected sides did not show a statistically significant benefit in 
terms of surgical field grade, surgery duration, blood loss, mean arterial pressure, $\mathrm{HR}$ or end-tidal $\mathrm{CO}_{2}$ (Table II).

In the regression analysis (univariate and multiple regression), with surgical field improvement as the outcome, and HR, mean arterial pressure or end-tidal $\mathrm{CO}_{2}$ as covariates, there was no correlation with any of the analysed variables.

There were no adverse events, either intra-operatively or post-operatively, related to pterygopalatine fossa injection. Only eight patients reported palatal discomfort post-operatively. No neurological side effects were recorded.

\section{Discussion}

The safety of FESS depends, in part, on a dry surgical field. Pterygopalatine fossa infiltration has been used in different procedures, including FESS, septorhinoplasty and dental regional anaesthesia, ${ }^{12}$ and for control of posterior epistaxis. ${ }^{13}$ It has been adopted routinely by some sinus surgeons but is not universally used.

In the current study, we wished to investigate the effect of the pterygopalatine fossa injection on the surgical field and blood loss during surgery. A previously described injection technique was used, ${ }^{10}$ which ensures optimal penetration through the greater palatine canal at its inferior aspect, with maximal vasospasm and minimal risk to the orbital contents, infraorbital nerve and maxillary artery.

We found no significant differences between the injected and non-injected sides in terms of the subjective outcome of surgical field grade ( $p=0.161)$, or the objective outcomes of blood loss $(p=0.630)$ and surgery duration $(p=0.994)$. Furthermore, there were no correlations between surgical field improvement and mean arterial pressure, $\mathrm{HR}$ or end-tidal $\mathrm{CO}_{2}$. This is in contrast to a previous well designed study

\begin{tabular}{lccc}
\multicolumn{4}{c}{ TABLE II } \\
\multicolumn{4}{c}{$\begin{array}{c}\text { MEAN SURGICAL FIELD GRADE AND MEASURED } \\
\text { VARIABLE VALUES BY TREATMENT GROUP }\end{array}$} \\
\hline Variable & $\begin{array}{c}\text { Injected side (mean } \\
\text { (SD)) }\end{array}$ & $\begin{array}{c}\text { Non-injected side } \\
\text { (mean (SD) })\end{array}$ & $p$ \\
\hline $\begin{array}{c}\text { Surgical } \\
\text { field } \\
\text { grade }\end{array}$ & $2.34(0.583)$ & $2.27(0.525)$ & 0.161 \\
$\begin{array}{c}\text { Surgery } \\
\text { duration } \\
\text { (min) }\end{array}$ & $38.67(20.713)$ & $37.80(20.923)$ & 0.994 \\
$\begin{array}{c}\text { Blood loss } \\
\text { (ml) }\end{array}$ & $165.07(141.548)$ & $153.62(117.882)$ & 0.630 \\
$\begin{array}{c}\text { MAP } \\
\text { (mmHg) }\end{array}$ & $70.91(10.014)$ & $70.50(9.410)$ & 0.495 \\
$\begin{array}{l}\text { HR (bpm) } \\
\text { EtCO } \\
(\mathrm{mmHg})\end{array}$ & $65.21(8.766)$ & $66.03(9.764)$ & 0.499 \\
\hline
\end{tabular}

$\mathrm{SD}=$ standard deviation; $\min =$ minutes $; \mathrm{MAP}=$ mean arterial pressure; $\mathrm{HR}=$ heart rate; $\mathrm{bpm}=$ beats per minute; $\mathrm{EtCO}_{2}=$ end-tidal carbon dioxide looking at the efficacy of adrenaline infiltration in the greater palatine canal to decrease intranasal bleeding during sinus surgery. ${ }^{14}$ That study, which was a prospective, blinded, randomised, controlled trial of pterygopalatine fossa injection, showed a significant difference in surgical field grade between the injected and non-injected sides in favour of the injected side $(p<0.01)$. Blood loss and surgery duration were not evaluated. We could not identify the possible cause of the difference in outcomes between that study and the current study.

Gurr et al. used laser Doppler blood flowmetry to measure the mucosal blood flow of the inferior turbinate before and after injection of the greater palatine canal. ${ }^{15}$ They found a 4.7 per cent decrease in blood flow to the inferior turbinate mucosa $(p=0.571)$ after the injection. While it is expected that adrenaline will cause vasoconstriction and a consequent decrease in blood flow, the clinical impacts of visual field bleeding and blood loss are unknown.

\section{- Control of bleeding during functional} endoscopic sinus surgery (FESS) is important for safety and efficiency

- Better visualisation during FESS helps minimise intra-operative and post-operative complications, and can reduce operative times

- Methods to reduce intra-operative bleeding include head elevation, bipolar cautery and microdebrider use, beta-blocker premedication, controlled hypotensive anaesthesia and total intravenous anaesthesia

- Pterygopalatine fossa infiltration prior to FESS did not improve intra-operative surgical field bleeding, but was not associated with any complications

Greater palatine canal injection with adrenaline may result in complications such as intravascular injection. Another potential side effect is infraorbital nerve injury and anaesthesia of the orbital nerves. These complications have been reported with a depth of penetration of $37 \mathrm{~mm} ;{ }^{16}$ in that study, infiltration was performed for regional anaesthesia for dental procedures. (In order to achieve reliable dental anaesthesia in such procedures, it is necessary to infiltrate close to the infraorbital nerve.) Therefore, the adrenaline injection for the purpose of vasoconstriction should be directed into the lower aspect of the pterygopalatine fossa at $25 \mathrm{~mm}$ depth, as used in this study. This is expected to produce vasospasm in the sphenopalatine artery while avoiding or significantly reducing the possibility of the above-mentioned complications.

\section{Conclusion}

Our results indicate that pterygopalatine fossa infiltration prior to FESS does not improve intra-operative 
surgical field bleeding, decrease blood loss or shorten surgery duration. The risks of the procedure, though minimal, are not warranted in the absence of significant benefit.

\section{References}

1 Stankiewicz JA. Complications of endoscopic sinus surgery. Otolaryngol Clin North Am 1989;22:749-58

2 Bolger WE, Kennedy DW. Surgical complications and postoperative care. In: Kennedy DW, Bolger WE, Zinreich SJ, eds. Diseases of the Sinuses: Diagnosis and Management. Hamilton, Ontario: PMPH USA, 2001;303-16

3 Hathorn IF, Habib AR, Manji J, Javer AR. Comparing the reverse Trendelenburg and horizontal position for endoscopic sinus surgery: a randomized controlled trial. Otolaryngol Head Neck Surg 2013;148:308-13

4 Eberhart LH, Folz BJ, Wulf H, Geldner G. Intravenous anesthesia provides optimal surgical conditions during microscopic and endoscopic sinus surgery. Laryngoscope 2003;113:1369-73

5 Nair S, Collins M, Hung P, Rees G, Close D, Wormald PJ. The effect of beta-blocker premedication on the surgical field during endoscopic sinus surgery. Laryngoscope 2004;114:1042-6

6 Pavlin JD, Colley PS, Weymuller EA Jr, Van Norman G, Gunn $\mathrm{HC}$, Koerschgen ME. Propofol versus isoflurane for endoscopic sinus surgery. Am J Otolaryngol 1999;20:96-101

7 Sieskiewicz A, Olszewska E, Rogowski M, Grycz E. Preoperative corticosteroid oral therapy and intraoperative bleeding during functional endoscopic sinus surgery in patients with severe nasal polyposis: a preliminary investigation. Ann Otol Rhinol Laryngol 2006;115:490-4

8 Wormald PJ, van Renen G, Perks J, Jones JA, Langton-Hewer $\mathrm{CD}$. The effect of the total intravenous anesthesia compared with inhalational anesthesia on the surgical field during endoscopic sinus surgery. Am J Rhinol 2005;19:514-20

9 Lund VJ, Kennedy DW. Staging for rhinosinusitis. Otolaryngol Head Neck Surg 1997;117:S35-40
10 Douglas R, Wormald PJ. Pterygopalatine fossa infiltration through the greater palatine foramen: where to bend the needle. Laryngoscope 2006;116:1255-7

11 Boezaart AP, van der Merwe J, Coetzee A. Comparison of sodium nitroprusside- and esmolol-induced controlled hypotension for functional endoscopic sinus surgery. Can J Anaesth 1995; $42: 373-6$

12 Lepere AJ. Maxillary nerve block via the greater palatine canal: new look at an old technique. Anesth Pain Control Dent 1993;2: 195-7

13 Bharadwaj VK, Novotny GM. Greater palatine canal injection: an alternative to the posterior nasal packing and arterial ligation in epistaxis. J Otolaryngol 1986;15:94-100

14 Wormald PJ, Athanasiadis T, Rees G, Robinson S. An evaluation of effect of pterygopalatine fossa injection with local anesthetic and adrenalin in the control of nasal bleeding during endoscopic sinus surgery. Am J Rhinol 2005;19:288-92

15 Gurr P, Callanan V, Baldwin D. Laser-Doppler blood flowmetry measurement of nasal mucosa blood flow after injection of the greater palatine canal. J Laryngol Otol 1996;110:124-8

16 Mercuri LG. Intraoral second division nerve block. Oral Surg Oral Med Oral Pathol 1979;47:109-13

Address for correspondence:

Dr Mark Samaha,

687 Pine Ave,

E-45,

Montreal,

Quebec H3A 1A1, Canada

Fax: +00 1 (514) 7315760

E-mail: mark_samaha@mac.com

Dr M Samaha takes responsibility for the integrity of the content of the paper

Competing interests: None declared 